\title{
Postmastectomy Pain Syndrome
}

\author{
Solmaz Fakhari ${ }^{1}$, Simin Atashkhoei ${ }^{2}$, Hojjat Pourfathi ${ }^{3}$, Haleh Farzin ${ }^{4}$, Eissa Bilehjani ${ }^{* *}$
}

\begin{abstract}
Breast cancer, recognized as the common cancer among women, is one of the important causes of morbidity and mortality worldwide. With regard to developments presented in screening, diagnosis, and especially therapies of this disease, survival of patients has improved in recent years, and as a result, the population at risk of complications attributable to treatment has increased, as well. Postmastectomy pain is one of the life-threatening complications of breast cancer. The nature of the mentioned pain is commonly neuropathic with abnormal sensation in intercostal nerve distribution region of upper and lateral parts of thorax, axilla, and medial and posterior parts of arm and shoulder. This pain strongly affects patients' quality of life. At present, the strongest theory about the etiology and mechanism of this phenomenon is related to the neuralgia of intercostobrachial nerve caused by the damage to the nerves after mastectomy. The type of surgery, especially complete axillary dissection (CAD), radiotherapy, chemotherapy, age, preand post-operative breast pain, type and size of tumor, all are considered as important risk factors. The best method for prevention and treatment of postmastectomy pain syndrome (PMPS) is multimodal approach. As the best treatment is prevention, may be prevention of this syndrome with consideration of the causative factors, timely resolution of these factors and improvement of surgery techniques could be achieved. Treatment approaches include both pharmacological interventions and non-pharmacological strategies. Pharmacotherapy is the major treatment of cancer-related pains. Intercostal nerve block, radiofrequency pulse, stellate ganglion block, neuromodulation techniques, intrathecal drug delivery, epidural injections of local anesthetic, mastopexy and use of botulinum, complementary and alternative therapies, scrambler therapy, acupuncture, yoga, and music therapy are the novel proposed strategies in this regard. Employing proper treatments can improve patients' quality of life.

Keywords: Postmastectomy pain, Breast cancer, Neuropathic Pain, Intercostobrachial nerve
\end{abstract}

\section{Introduction}

Breast cancer is a common cancer-affecting women. It is an important cause of female morbidity and mortality worldwide and accounts for more than $30 \%$ of all new cases of cancer in females (1). Prevalence of this cancer is varying in different countries, which results from many factors such as age, race, socioeconomic status, lifestyle, reproductive history, family history, etc $(2,3)$.

With the exception of Japan, breast cancer has higher incidence rate in developed countries in comparison with developing countries. The National Cancer Institution-Cairo estimates that $12.7 \%$ of women are exposed to breast cancer diagnosis over their lifetime. Currently, $80 \%$ of patients have presented a 5 -year survival due to early diagnosis and improvements of management in this regard (1,4-6). Developments in screening, diagnosis, and especially innovative therapies have positive effects on reducing mortality rate, which ultimately help patients frequently survive several years. For instance, age-standardized mortality rate in 1977 equated to 30.6 deaths per 100000 population, whereas it reached to 24.6 deaths per 100000 population in 2002 (2, 7-10). Considering decreased breast cancer-related mortality rate, the population at risk of complications attributable to treatment of this disease, especially after mastectomy, has increased $(7,9)$. As treatment of breast cancer usually starts with surgery, approximately $60 \%$ of patients undergo surgery for primary tumor resection, axillary node staging, or mastectomy $(8,10)$.

The side effects associated with breast cancer treatment can occur in about $90 \%$ of patients. These complications may continue for months or years after treatment, having many implications on survivors' quality of life (5). One of the life-threatening complications is occurrence of chronic pain following surgical treatment, which is known as postmastectomy pain syndrome (PMPS) (11). The present study aimed at reviewing symptoms, risk factors, etiology, prevalence rate, prevention, and treatment methods in this regard.

\section{Epidemiology}

PMPS is a distinctive syndrome with chronic and debilitating neuropathic pain, which can develop after cancer surgery including radical mastectomy, modified radical

Received 3 September 2016, Accepted 23 December 2016, Available online 1 January 2017

${ }^{1}$ Assistant Professor of Anesthesiology, Fellowship in Palliative Medicine, Tabriz University of Medical Sciences, Tabriz, Iran. ${ }^{2}$ Professor of Anesthesiology, Al-Zahra Hospital, Tabriz University of Medical Sciences, Tabriz, Iran. ${ }^{3}$ Assistant Professor of Anesthesiology, Taleghani Hospital, Tabriz University of Medical Sciences, Tabriz, Iran. ${ }^{4}$ Assistant of Anesthesiology. Tabriz University of Medical Sciences, Tabriz, Iran. ${ }^{5}$ Assistant Professor of Cardiac Anesthesiology, Madani Heart Center, Tabriz University of Medical Sciences, Tabriz, iran.

*Corresponding Author: Eissa Bilehjani, Tel: +989141160647; Email: isadavod@gmail.com 
mastectomy, and segmental mastectomy (lumpectomy) associated with axillary lymph node dissection $(3,12,13)$. It is estimated that almost $25 \%-60 \%$ of patients suffer from chronic pain after breast cancer-related surgery (11). Wood first reported this pain in late 1970, and then Folly et al described PMPS consisting of pain and sensory disorders following mastectomy (8).

\section{Etiology and Mechanism}

Pathophysiologic Mechanism

Exact etiology and mechanism involved in PMPS are indeterminate although it seems to be probably multifactorial $(3,14)$. The nature of pain is commonly chronic neuropathic, which results from nociceptive axonal injury $(3,10)$.

It is believed that common cause may be neural pathway damage, particularly damage of intercostobrachial nerve, which is the cutaneous lateral branch of T2, during the surgery performed on breast and axillary area. Cutaneous lateral T2 branch (intercostobrachial) supplies supper part of thorax, upper and internal portion of arm and passes through axilla $(3,15)$. Axillary dissection is associated with intercostobrachial nerve damage due to stretching during retraction or frank transection (10). One study indicated that in spite of maintaining brachial plexus and peripheral nerves, the damage caused by compression or pressure could result in PMPS. Another study reported that $80 \%-100 \%$ of patients undergoing mastectomy and axillary dissection are affected by intercostobrachial nerve damage (16).

Symptoms can usually occur in nerve distribution area; however, the most common theory for PMPS onset is sensory type of damage to intercostobrachial nerve $(3,15)$. One neurologic study performed on patients with PMPS demonstrated that intercostobrachial nerve damage can occur in almost all patients (16). It is obvious that the type of breast surgery influences the prevalence of PMPS. It appears that prevalence of PMPS following breast conservation treatment is higher than its prevalence following modified radical mastectomy. Lower prevalence has been observed after sentinel node biopsy, which can be explained by invasive nature of complete axillary dissection (CAD) in comparison with sentinel node biopsies (SLND), recognized as the latest technique removing only cancer draining lymph node. In other words, there is good chance for preservation of nerve and vasculature in this situation (15-18). Post-simple mastectomy and laminectomy PMPS can usually occur due to injury to internal branch of intercostobrachial.

\section{Risk Factors}

It has been acknowledged that radiotherapy aggravates surgery-induced injury (15) . Gerber et al in their study indicated higher prevalence of PMPS after one to even six years following lumpectomy associated with radiotherapy (19). However, radiotherapy can result in further damages to intercostobrachial and lead to exacerbation of PMPS. In addition, chemotherapy drugs, e.g. taxol, vincristine, and platinum, and lymph edema affect pain severity and discomfort caused by operation $(15,20)$. In patients suffering from chronic neuropathic pain, alteration in CNS pain-modulatory mechanism and remodeling of cerebral portions that account for processing of painful stimulations can be considered as the possible reasons. In these patients, limbic system is hypersensitized to painful stimulations and leads to development of feedback between damaged sensory nerves and excitation pain center in brain $(15,21)$.

Various studies have considered the association between adjuvant cancer therapy and PMPS. Carpenter et al. reported high prevalence of PMPS among women undergoing lumpectomy and chemo-radiotherapy (22).

Other risk factors include prior history of headache, being younger than 50 years old (especially 35 years old), genetic sensibility, psychosocial situation, marital status, employment status, housing, presence of post-operative pain, smoking, and presence of pain during peri-operative period (23-25). It seems that tumor in younger patients is negative estrogen-response and high grade. Furthermore, the type of tumor is proliferative and frequently induces vascular invasion $(10,11)$. In one study, symptoms of PMPS were shown in $65 \%$ of patients within the age range of $30-45$, whereas the rate was $26 \%$ in women aged 70 and over (15).

The larger size of tumor, post-operative complications (e.g. infection and bleeding), pre-operative depression, anxiety, and psychological stress play probably important roles in development of this syndrome $(10,11,26)$. One study reported that PMPS is more common in patients with previous history of breast surgery and in patients with their tumor located in the upper lateral quarter (27). El-Sayed anf Ali found an association between educational level and dimensions of quality of life score. Patients with lower educational level had better physical and psychological status (1). Other studies stated that over use of arm $(62 \%)$, straining (42\%), sudden movements (38\%), cold (16\%), stress and edema (14\%), cough (13\%), immobility, arm compression, and prolonged standing (11\%) can exacerbate symptoms of PMPS. Overall, incidence of PMPS varies between $20 \%-50 \%$ in the conducted studies $(9,18,22)$.

\section{Symptoms}

PMPS is a chronic neuropathic pain that typically occurs as a continuous pain in association with abnormal sensation in inter-costal nerve distribution region of upper and lateral parts of thorax, axilla, and medial and posterior parts of arm and shoulder. Symptoms are shock-like with tingling pain sensation and burning pain related to chronic dysesthesia or shooting pain, pressure sensation, numbness, or pricking $(10,12,18,22)$. Pain on ipsilateral site of surgery can occur in anterior part of chest, axilla, and mid and upper parts of arm and shoulder. Pain may be present at least 4 days per week or may last for longer than 3 months. According to IASP definition, pain sustains over normal adequate duration to allow healing. Based on nu- 
merical rating scale (NRS), it has an average intensity of at least 3 on a scale from 0 to $10(7,9,11,23,28)$. Chronic neuropathic pain related to PMPS with enough severity can interfere with sleep and daily activity performance, and poor treatment can result in immobilized patient or sever lymph edema, frozen shoulder syndrome, and complex regional pain (CRPS) (2).

Pain typically occurs immediately after post-operative period; however, it may initiate with a delay of 6 months and longer after surgery and last for years (11). According to Miguel et al, incidence of pain was reported as follow: axillary pain (84\%), upper part of arm pain $(74 \%)$, pin in upper part of chest pain (58\%), and shoulder pain (32\%) (12).

\section{Quality of Life}

PMPS neuropathic pain has major impacts on patients' health, function, and quality of life. Chronic pain has interferences with physical and occupational functions, mood and sleep, and relationships and enjoyment of life. In $50 \%$ of patients, pain interferes with daily activity and may lead to sleep disturbances (11).

However, some interventions are required to improve patients' symptoms, quality of life, and functional capacity (3).

\section{Prognosis}

This chronic pain syndrome has been frequently underestimated and poorly managed (12); however, in a number of studies, it was indicated that its intensity and associated sensory abnormalities diminished over time (10).

\section{Prevention}

Improvement of Technique

Although protection of intercostobrachial nerve is clinically difficult in practice, careful protection or dissection may decrease occurrence of sensory deficits and intercostobrachial neuralgia (ICN). SLND, which is preferred to $\mathrm{CDA}$, is a better technique that can decrease sensory deficits and ICN. Hence, CDA is only performed in lymphatic node involvement cases $(9,10)$.

\section{Alleviation of Risk Factors}

Severe acute pre-operative or post-operative pain is suggested as a contributing risk factor for some pain syndromes such as PMPS. It is recommended that acute pain should be appropriately managed to decrease post-operative chronic pain (10). Strazisar et al addressed the role of acute pain in post-operative chronic pain and showed positive impact of continuous infusion of local anesthetic by placing catheter on mastectomy surgery incision site (24). Moreover, Fassoulaki et al demonstrated that in patients undergoing mastectomy or lumpectomy in combination with axillary lymph node dissection, in which eutectic mixture of local anesthetic (EMLA) was used during pre-operative period for 3 days after surgery, chronic pain occurrence may be reduced 3 months after operation (29). Lakdja et al revealed beneficial effects of pre-operative administration of prophylactic Nonsteroidal anti-inflammatory drugs (NSAIDs) in PMPS, even 6 months after mastectomy (20).

However, based on inadequate evidence presented in this regard, objectives of prophylactic strategy must primarily include optimization of pain control during pre-operative period and decrease of nerve injury during surgery. In addition to EMLA, drugs such as gabapantine and mexiletine are found to be useful in patients undergoing breast surgery to reduce their post-operative acute and chronic pain $(29,30)$. Reduction of surgery-related injury could be accompanied by improvement of operation methods and techniques effective in pain relief. In addition, improvement of screening methods for early diagnosis of breast cancer is another important factor in PMPS relief. Early detection is equivalent with smaller size of tumor, which in turn results in minimally invasive surgical treatment and facilitates utilization of breast cancer conservative techniques such as lumpectomy, breast-conserving surgery, extensive local excision, partial mastectomy, segmentectomy, or tylectomy $(9,11,31)$. Even so, increased application of staging techniques with minimal invasion such as sentinel lymph node biopsy may decrease axillary dissection (32). In one study, paravertebral thoracic nerve block was used to reduce post-operative chronic and acute pains (33).

\section{Treatment}

According to the World Health Organization (WHO), pharmacotherapy is the major treatment of cancer-related pain. Based on the WHO analgesic ladder (Table 1), treatments proposed for cancer pain must follow sequential order, which is initiated by NSAIDs, and if analgesia is not sufficient, different types of opioids should be administered.

Adjunct drugs at any stages of WHO ladder can be added to the main drugs. Antidepressant agents such as gabapentinoids (e.g. gabapentin, pregabalin), antiepileptic drugs, narcoleptic drugs (e.g. chlorpromazine), serotonin reuptake inhibitors (e.g. fluoxetine, venlafaxine), NMDA antagonists (e.g. ketamine, memantine), lidocaine patch $5 \%$, and short-term administration of steroids are used for treating chronic neuropathic pain (34).

The best method for prevention and treatment of PMPS is multimodal approach. The most appropriate treatment is prevention, which could be somewhat performed by application of and close attention to nerve preservation during surgery, using minimally invasive procedures and SLND rather than CAD.

Using EMLA $25 \mathrm{mg}$ lidocaine and $25 \mathrm{mg}$ prilocaine in $1 \mathrm{~mL}$ water in pre-operative period can decrease the need to post-operative analgesia and can reduce the possible occurrence of post-operative chronic neuropathic pain (15). Dini et al in their investigation showed the beneficial effects of capsaicin $0.025 \%$ in this regard (35). Capsaicin (Qutenza) 8\% (concentrated extract of chili peppers) is an interesting and more potent drug in comparison with capsaicin $0.025 \%$ and is usually effective (sometimes works 
Table 1. Three-Step Analgesic Ladder Based on the World Health Organization (WHO)

\begin{tabular}{lll}
\hline Mild Pain & $\begin{array}{l}\text { Moderate Pain (or Mild Pain Unrelieved } \\
\text { by Previous Step) }\end{array}$ & $\begin{array}{l}\text { Severe Pain (or Mild to Moderate Pain } \\
\text { Unrelieved by Previous Step) }\end{array}$ \\
\hline Non-opioid analgesics: & Opioid analgesics for step2: & Opioid analgesics for step3: \\
Acetaminophen, ibuprofen, naproxen & Codeine, oxycodone, hydrocodone, & Higher doses of morphine, fentanyl, \\
+/- Adjuvants: & morphine & hydromorphine, PCA delivery of IV opioid \\
Anticonvulsants for neuropathic pain & + +- Non-opioid analgesics: & + /- Non-opioid analgesics: \\
And antidepressants or anxiolytics for coexisting & (from previous step) & (from previous step) \\
mood disturbances & $+/-$ Adjuvant: & + /-Adjuvants: \\
& (from previous step) & (from previous step) \\
\hline
\end{tabular}

where nothing else works) (36). Wagner et al addressed the effect of inter-costal nerve blocks with local anesthetic and corticosteroids on ICN injuries and indicated the positive response to this approach; he recommended radiofrequency pulse (36). Stellate ganglion block is another recommended therapy for PMPS that results in cervical sympathetic trunk block or vertebral ganglion, postganglionic and pre-ganglionic fibers, inferior cervical sympathetic ganglia, and thoracic sympathetic ganglia $(13,37,38)$. Neuromodulation techniques including stimulation of brain motor cortex, SCS (spinal cord stimulation), intrathecal drug delivery, and epidural injection of local anesthetic are used to relieve PMPS (39-41). Application of complement therapy or alternative therapy has increased for improvement of health. Utilization of these treatments in patients with breast cancer is higher in comparison with other populations that suffer from other types of cancer. By clarification of objective and subjective elements of cancer pain, patients may adopt and take advantage of these cognitive behavioral therapies, e.g. progressive muscle relaxation training, hypnosis, distraction, to reduce breast pain (6). In addition, guided imagery can be employed to modulate pain and alter transmission and perception of painful stimulus by distracting patients' attention (6). Dessy et al assessed the role of mastopexy and botulinum on treatment of PMPS (42). Toxin of botulinum may inhibit the release of neurotransmitters contributing to impulse transmission. These neurotransmitters include endothelin I, substance P, CGRP, and neuropeptide $\mathrm{Y}$. Crew et al showed the positive effects of acupoint stimulation, electropuncture, acupuncture, and acupressure. Anti-nociceptive effects of acupuncture result from several mechanisms such as discharge of polymodal receptor, increased circulating endogenous opioid peptides level, improvement of circulation, and mechanotransduction-based responses (43). Scrambler therapy is an electro-analgesic technique for regulating pain and is effective for improving neuropathic pain and probably PMPS (44). Yoga has positive implications for behavioral outcome, for example, pain, depression, mood, and quality of life, and it may be useful in treatment of PMPS (45). Li et al reported that music therapy is significantly effective on reducing PMPS (46).

\section{Summary}

Improvement of the survival rate of patients with breast cancer results in increased incidence rate of PMPS. There is in fact limited data on causality and risk factors of PMPS. Treatment approaches include both pharmacological interventions and non-pharmacological strategies. However, current treatments of the PMPS are sub-optimal. Further investigations are required to achieve the appropriate developments in diagnosis and screening of breast cancer, and evaluation and treatment of PMPS that will provide less side effects, adequate analgesia, and finally, improved quality of life, and patient satisfaction in the future.

\section{Conclusion}

Frequency of PMPS due to advances in diagnosis and treatment of breast cancer is on the rise. Using proper treatments can improve the patients' quality of life.

\section{Ethical Issues}

Not applicable.

\section{Conflict of Interests}

The authors have no conflicts of interest to disclose.

\section{Financial Support}

None.

\section{References}

1. El-Sayed NO, Ali ZH. Effect of counseling intervention post mastectomy for women undergoing adjuvant chemotherapy on their quality of life. J Cancer Sci Ther. 2011;3:7. doi:10.4172/1948-5956.S1.19.

2. Variawa ML. The prevalence of chronic postmastectomy pain syndrome in female breast cancer survivors. Southern African Journal of Anaesthesia and Analgesia. 2016;22(4):108-113. doi:10.1080/22201181.2016.1191214.

3. Dey S, Soliman AS, Hablas A, et al. Urban-rural differences in breast cancer incidence by hormone receptor status across 6 years in Egypt. Breast Cancer Res Treat. 2010;120(1):149160. doi:10.1007/s10549-009-0427-9

4. Omar S, Khaled H, Gaafar R, Zekry A, Eissa S, El Khatib O. Breast cancer in Egypt: a review of disease presentation and detection strategies. East Mediterr Health J. 2003;9(3):448463.

5. Smith HS, Wu S-X. Persistent pain after breast cancer treatment. Ann Palliat Med. 2013;1(3):182-194.

6. Satija A, Ahmed SM, Gupta R, et al. Breast cancer pain management - A review of current \& novel therapies. Indian J Med Res. 2014;139(2):216.

7. Narod SA, Iqbal J, Miller AB. Why have breast cancer mortality rates declined? JCancer Policy. 2015;5:8-17. 
8. Bokhari F, Sawatzky J-AV. Chronic neuropathic pain in women after breast cancer treatment. Pain Manag Nurs. 2009;10(4):197-205.

9. Shukla NK, Bhatnagar S. Phantom breast syndrome. Indian J Palliat Care. 2009;15(2):103.

10. Jung BF, Ahrendt GM, Oaklander AL, Dworkin RH. Neuropathic pain following breast cancer surgery: proposed classification and research update. Pain. 2003;104(1-2):113.

11. Mejdahl MK, Andersen KG, Gärtner R, Kroman N, Kehlet H. Persistent pain and sensory disturbances after treatment for breast cancer: six year nationwide follow-up study. BMJ. 2013;346:f1865. doi:10.1136/bmj.f1865.

12. Miguel R, Kuhn AM, Shons A, et al. The effect of sentinel node selective axillary lymphadenectomy on the incidence of postmastectomy pain syndrome. Cancer Control. 2001;8(5):427-430.

13. Abdel Dayem OT, Saeid MM, Ismail OM, El Badrawy AM, Abdel Ghaffar NA. Ultrasound guided stellate ganglion block in postmastectomy pain syndrome: a comparison of ketamine versus morphine as adjuvant to bupivacaine. Journal of Anesthesiology. 2014;2014:792569.

14. Meijuan Y, Zhiyou P, Yuwen T, Ying F, Xinzhong C. A retrospective study of postmastectomy pain syndrome: incidence, characteristics, risk factors, and influence on quality of life. ScientificWorldJournal. 2013;2013:159732. doi:10.1155/2013/159732.

15. Selim S, Shapiro R, Hwang E, Rosenbauni E. Post breast therapy pain syndrome (PBTPS). http://www. cancersupportivecare.com/neuropathicpain.php. Published June 1, 2002.

16. Vecht C, Van de Brand H, Wajer O. Post-axillary dissection pain in breast cancer due to a lesion of the intercostobrachial nerve. Pain. 1989;38(2):171-176.

17. Cody HS. Sentinel lymph node mapping in breast cancer. Breast Cancer. 1999;6(1):13-22.

18. Warmuth MA, Bowen G, Prosnitz LR, et al. Complications of axillary lymph node dissection for carcinoma of the breast. Cancer. 1998;83(7):1362-1368.

19. Gerber L, Lampert M, Wood C, et al. Comparison of pain, motion, and edema after modified radical mastectomy vs. local excision with axillary dissection and radiation. Breast Cancer Res Treat. 1992;21(2):139-145.

20. Lakdja F, Dixmerias F, Bussieres E, Fonrouge J, Lobera A. [Preventive analgesic effect of intraoperative administration of ibuprofen-arginine on postmastectomy pain syndrome]. Bull Cancer. 1997;84(3):259-63.

21. Edwards RR, Mensing G, Cahalan C, et al. Alteration in pain modulation in women with persistent pain after lumpectomy: influence of catastrophizing. J Pain Symptom Manage. 2013;46(1):30-42. doi:10.1016/j. jpainsymman.2012.06.016.

22. Carpenter JS, Andrykowski MA, Sloan P, et al. Postmastectomy/postlumpectomy pain in breast cancer survivors. J Clin Epidemiol. 1998;51(12):1285-1292.

23. de Menezes Couceiro TC, Valença MM, Raposo MCF, de Orange FA, Amorim MM. Prevalence of post-mastectomy pain syndrome and associated risk factors: a cross-sectional cohort study. Pain Manag Nurs. 2014;15(4):731-737. doi: 10.1016/j.pmn.2013.07.011

24. Strazisar B, Besic N. Comparison of continuous local anaesthetic and systemic pain treatment after axillary lymphadenectomy in breast carcinoma patients-a prospective randomized study. Radiol Oncol. 2013;47(2):145-153.

25. Wang L, Guyatt GH, Kennedy SA, et al. Predictors of persistent pain after breast cancer surgery: a systematic review and meta-analysis of observational studies. CMAJ. 2016;188(14):E352-E361.

26. Cox CE, Haddad F, Bass S, et al. Lymphatic mapping in the treatment of breast cancer. Oncology (Williston Park, NY). 1998;12(9):1283-1292.

27. Vilholm OJ, Cold S, Rasmussen La, Sindrup SH. The postmastectomy pain syndrome: an epidemiological study on the prevalence of chronic pain after surgery for breast cancer. Br J Cancer. 2008;99(4):604-610.

28. Foley KM. Pain syndromes in patients with cancer. Med Clin North Am. 1987;71(2):169-84.

29. Fassoulaki A, Sarantopoulos C, Melemeni A, Hogan Q. EMLA reduces acute and chronic pain after breast surgery for cancer. Reg Anesth Pain Med. 2000;25(4):350-355.

30. Fassoulaki A, Patris K, Sarantopoulos C, Hogan Q. The analgesic effect of gabapentin and mexiletine after breast surgery for cancer. Anesth Analg. 2002;95(4):985-991.

31. Rietman J, Geertzen J, Hoekstra H, et al. Long term treatment related upper limb morbidity and quality of life after sentinel lymph node biopsy for stage I or II breast cancer. Eur J Surg Oncol. 2006;32(2):148-152.

32. Peintinger F, Reitsamer R, Stranzl H, Ralph G. Comparison of quality of life and arm complaints after axillary lymph node dissection vs sentinel lymph node biopsy in breast cancer patients. Br J Cancer. 2003;89(4):648-652.

33. Ilfeld BM, Madison SJ, Suresh PJ, et al. Treatment of postmastectomy pain with ambulatory continuous paravertebral nerve blocks: a randomized, triple-masked, placebocontrolled study. Reg Anesth Pain Med. 2014;39(2):89.

34. Vadalouca A, Raptis E, Moka E, Zis P, Sykioti P, Siafaka I. Pharmacological treatment of neuropathic cancer pain: a comprehensive review of the current literature. Pain Pract. 2012;12(3):219-251.

35. Dini D, Bertelli G, Gozza A, Forno GG. Treatment of the post-mastectomy pain syndrome with topical capsaicin. Pain. 1993;54(2):223-226.

36. Wagner T, Poole C, Roth-Daniek A. The capsaicin $8 \%$ patch for neuropathic pain in clinical practice: a retrospective analysis. Pain Med. 2013;14(8):1202-1211. doi: 10.1111/ pme.12143.

37. Aghamohamadi D, Hosseinzadeh $\mathrm{H}$, Golzari S, et al. Preincisional ipsilateral stellate ganglion block for acute post operative pain control in unilateral mastectomy. Pak J Med Sc 2011;27(4):879-883.

38. Elias M. Cervical sympathetic and stellate ganglion blocks. Pain Physician. 2000;3(3):294-304.

39. Brown JA, Pilitsis JG. Motor cortex stimulation. Pain Med. 2006;7(s1):S140-S145.

40. Nuti C, Peyron R, Garcia-Larrea L, et al. Motor cortex stimulation for refractory neuropathic pain: four year outcome and predictors of efficacy. Pain. 2005;118(1):4352 .

41. De Andrés J, Buyten V. Neural modulation by stimulation. Pain Pract. 2006;6(1):39-45.

42. Dessy L, Maruccia M, Mazzocchi M, Scuderi N. Treatment of post mastectomy pain syndrome after mastopexy with botulinum toxin. J Plast Reconstr Aesthet Surg. 2014;67(6):873-874. doi: 10.1016/j.bjps.2013.12.032.

43. Crew KD, Capodice JL, Greenlee H, et al. Randomized, 
blinded, sham-controlled trial of acupuncture for the management of aromatase inhibitor-associated joint symptoms in women with early-stage breast cancer. J Clin Oncol. 2010;28(7):1154-1160.

44. Marineo G, Iorno V, Gandini C, Moschini V, Smith TJ. Scrambler therapy may relieve chronic neuropathic pain more effectively than guideline-based drug management: results of a pilot, randomized, controlled trial. J Pain Symptom Manage. 2012;43(1):87-95. doi: 10.1016/j. jpainsymman.2011.03.015.
45. Galantino ML, Greene L, Archetto B, et al. A qualitative exploration of the impact of yoga on breast cancer survivors with aromatase inhibitor-associated arthralgias. Explore (NY). 2012;8(1):40-7. doi: 10.1016/j.explore.2011.10.002.

46. Li X-M, Yan H, Zhou K-N, Dang S-N, Wang D-L, Zhang Y-P. Effects of music therapy on pain among female breast cancer patients after radical mastectomy: results from a randomized controlled trial. Breast Cancer Res Treat. 2011;128(2):411-419. doi: 10.1007/s10549-011-1533-z.

Copyright (c) 2017 The Author(s); This is an open-access article distributed under the terms of the Creative Commons Attribution License (http://creativecommons.org/licenses/by/4.0), which permits unrestricted use, distribution, and reproduction in any medium, provided the original work is properly cited. 